\title{
Fast Mesh Refinement in Pseudospectral Optimal Control
}

\author{
N. Koeppen*, I. M. Ross ${ }^{\dagger}$, L. C. Wilcox ${ }^{\ddagger}$, R. J. Proulx ${ }^{\S}$ \\ Naval Postgraduate School, Monterey, CA 93943
}

\begin{abstract}
Mesh refinement in pseudospectral (PS) optimal control is embarrassingly easy simply increase the order $N$ of the Lagrange interpolating polynomial and the mathematics of convergence automates the distribution of the grid points. Unfortunately, as $N$ increases, the condition number of the resulting linear algebra increases as $N^{2}$; hence, spectral efficiency and accuracy are lost in practice. In this paper, we advance Birkhoff interpolation concepts over an arbitrary grid to generate well-conditioned PS optimal control discretizations. We show that the condition number increases only as $\sqrt{N}$ in general, but is independent of $N$ for the special case of one of the boundary points being fixed. Hence, spectral accuracy and efficiency are maintained as $N$ increases. The effectiveness of the resulting fast mesh refinement strategy is demonstrated by using polynomials of over a thousandth order to solve a low-thrust, long-duration orbit transfer problem.
\end{abstract}

\section{Introduction}

In principle, mesh refinement in pseudospectral (PS) optimal control[1] is embarrassingly easy - simply increase the order $N$ of the Lagrange interpolating polynomial. For instance, in a Chebyshev PS method[2, 3, 4], the Chebyshev-Gauss-Lobatto (CGL) mesh points are given by[2],

$$
t_{i}=\frac{1}{2}\left[\left(t_{f}+t_{0}\right)-\left(t_{f}-t_{0}\right) \cos \left(\frac{i \pi}{N}\right)\right], \quad i=0,1, \ldots, N
$$

where, $\left[t_{0}, t_{f}\right]$ is the time interval. Grid points generated by (1) for $N=5,10$ and 20 over a canonical time-interval of $[0,1]$ are shown in Fig. 1. The production of these mesh points is as fast as it takes to compute the cosine function. Furthermore, the mesh points are at optimal locations for a Chebyshev PS method because their placement is dictated by well-established rules of approximation theory[5]. The price for this speed and simplicity is an increase in the condition number of the underlying linear algebra that governs the iterations[1, 2, 6]. In fact, as shown in Fig. 2, the condition number associated with the PS discretization of the dynamical equations,

$$
\dot{\boldsymbol{x}}(t)=\boldsymbol{f}(\boldsymbol{x}(t), \boldsymbol{u}(t), t)
$$

${ }^{*}$ Graduate Student and Captain, USMC, Control and Optimization Laboratories, Department of Mechanical and Aerospace Engineering

${ }^{\dagger}$ Distinguished Professor and Program Director, Control and Optimization, Department of Mechanical and Aerospace Engineering

*Associate Professor, Department of Applied Mathematics

${ }^{\S}$ Research Professor, Control and Optimization Laboratories, Space Systems Academic Group 


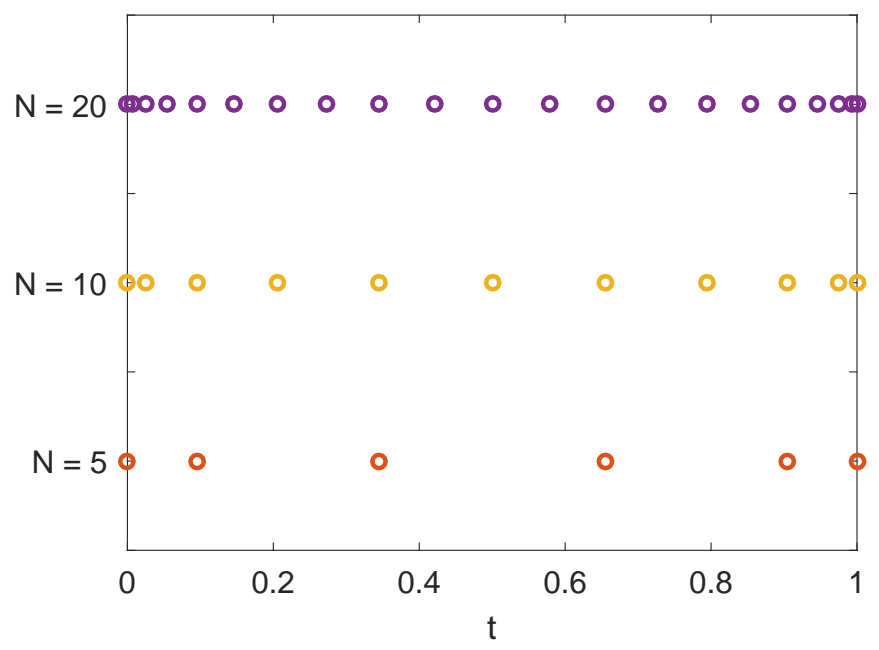

Figure 1. Grid points for $N=5,10$ and 20 for a Chebyshev pseudospectral method.

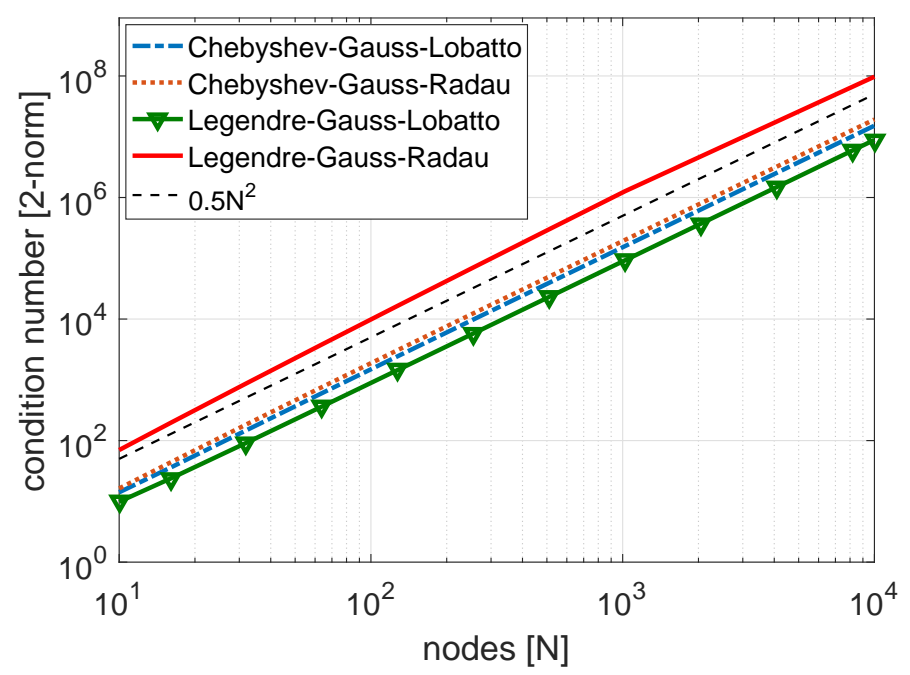

Figure 2. Illustrating $\mathcal{O}\left(N^{2}\right)$-growth in the condition number associated with (2) for various mesh selections.

increases as $N^{2}$. The plots in this figure are the condition numbers of the non-singular, inner, square-portion of the various differentiation matrices[1,2] associated with the indicated mesh selections. Note that the Legendre-Gauss-Radau mesh has the worst performance of all the indicated PS grid selections. An intuitive approach to manage the growth in the condition number is to break up the time interval into several segments and apply a PS method over each segment $[7,8,9,10]$. When the segments are non-overlapping and information across the segments is passed across a single point, it results in a PS knotting method[8, 10]; see Fig. 3. The special nodes where information across segments is transmitted are called PS knots. In the spectral algorithm for PS methods[10,11], the number and location of the PS knots are determined adaptively. The adaptation process is based on an analysis of the solution generated by the prior iterate. Among other things, the analysis involves an estimation of the error and a determination 


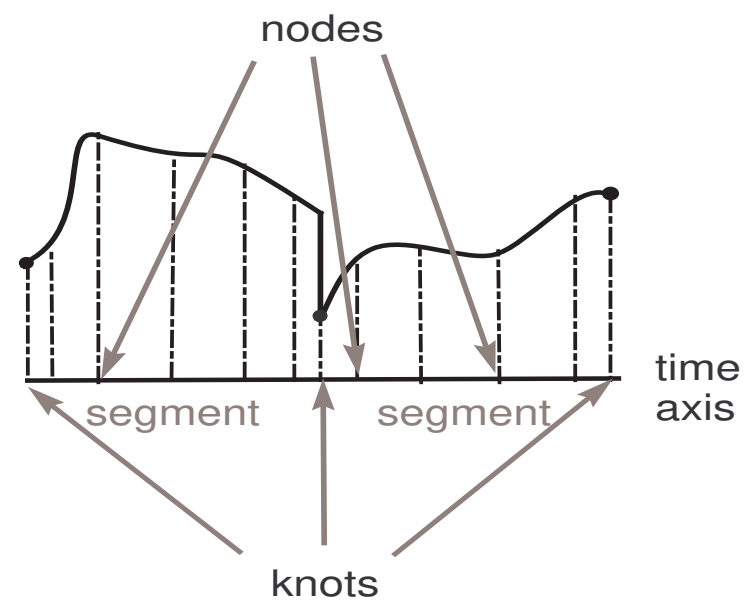

Figure 3. An illustration of PS knots in a generic pseudospectral knotting method; adapted from [8].

of the frequency content of the system trajectory. Based on this analysis, either $N$ per segment is increased or new knots are placed near high frequency regimes[10,11]. The entire process is repeated for the next iterate or until all stopping criteria are met[1, 8, 10, 11].

Although the PS knotting method manages the growth in the condition number by simply limiting the order of the approximating polynomial in a given segment, the price for this approach is quite severe. First, there is an increase in the computation time to determine the number and location of the knots; second, there is a significant reduction in the rate of convergence[12, 13, 14, 15] that adds to the increase in computational time and effort. The convergence rate varies inversely to the number of segments - the more the segments, the poorer the convergence rate. If the order of the approximating polynomial is limited to low orders (e.g., $N=4$ ), a PS method becomes theoretically equivalent to a Runge-Kutta method[16, 17, 18]. In fact, the "exponential" rate of convergence of an unknotted PS method is chiefly due to the high orders (i.e., $N \gg 10$ ) of the approximating polynomials[2, 5]. In many aerospace applications[19, 20, 21, 22], including flight implementations[1, 23, 24, 25, 26, 27, 28], very accurate solutions[29, 30] are generated for $N \leq 100$. In certain emerging areas of applications[31, 32, 33], there have been new requirements to generate solutions for $N \gg 100$. Because the unknotted PS method generates high condition numbers, various types of preconditioning are often used to alleviate the problem[34, 35]. In recent years, there have been major advancements in well-conditioned PS methods[36, 37, 38] for ordinary differential equations. These methods offer a remarkable drop in the condition number from $\mathcal{O}\left(N^{2}\right)$ to $\mathcal{O}(1)$. In this paper, we adapt these advancements for PS optimal control using Birkhoff interpolants[36]. The dramatic drop in the condition number allows us to generate solutions for thousands of grid points (i.e., using approximating polynomials for state trajectories in excess of a thousand degrees) thereby generating a very fast mesh refinement strategy. Numerical results for a sample low thrust trajectory optimization problem are presented to illustrate the advancements.

The citation for the journal version of this paper is: J. Guid., Contr. \& Dyn., 42/4, 2019, pp. 711-722. 


\section{A Brief Review of the State-of-the-Art in PS Optimal Control Methods}

In order to provide a proper context for the issues in PS optimal control methods, we first briefly review the state of the art. To this end, consider a generic optimal control problem given by[39, 40, 41],

$$
\begin{aligned}
& \mathbb{X}=\mathbb{R}^{N_{x}} \quad \mathbb{U}=\mathbb{R}^{N_{u}} \\
& \boldsymbol{x}=\left(x_{1}, \ldots, x_{N_{x}}\right) \quad \boldsymbol{u}=\left(u_{1}, \ldots, u_{N_{u}}\right) \\
& \text { problem }\left\{\begin{array}{rr}
\text { Minimize } & J\left[\boldsymbol{x}(\cdot), \boldsymbol{u}(\cdot), t_{0}, t_{f}\right]:= \\
& E\left(\boldsymbol{x}_{0}, \boldsymbol{x}_{f}, t_{0}, t_{f}\right)+\int_{t_{0}}^{t_{f}} F(\boldsymbol{x}(t), \boldsymbol{u}(t), t) d t
\end{array}\right\} \text { (cost) }
\end{aligned}
$$

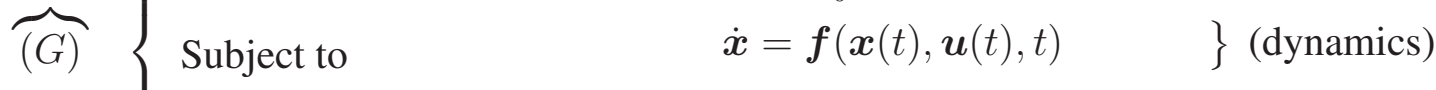

$$
\begin{aligned}
& \left.\boldsymbol{e}^{L} \leq \boldsymbol{e}\left(\boldsymbol{x}_{0}, \boldsymbol{x}_{f}, t_{0}, t_{f}\right) \leq \boldsymbol{e}^{U}\right\} \text { (events) } \\
& \left.\boldsymbol{h}^{L} \leq \boldsymbol{h}(\boldsymbol{x}(t), \boldsymbol{u}(t), t) \leq \boldsymbol{h}^{U} \quad\right\} \text { (path) }
\end{aligned}
$$

where, the symbols denote the following:

- $\mathbb{X}$ and $\mathbb{U}$ are $N_{x^{-}}$and $N_{u}$-dimensional real-valued state and control spaces respectively. We assume $N_{x} \in \mathbb{N}^{+}$and $N_{u} \in \mathbb{N}^{+}$.

- $J$ is the scalar cost function. The arguments of $J$ are the optimization variables.

- The optimization variables are:

- $\boldsymbol{x}(\cdot)$ : the $N_{x}$-dimensional state trajectory,

- $\boldsymbol{u}(\cdot)$ : the $N_{u}$-dimensional control trajectory,

- $t_{0}$ : the initial clock time, and

$-t_{f}:$ the final clock time.

- $E$ is the scalar endpoint cost function. The arguments of $E$ are the endpoints. In the classical literature, $E$ is known as the "Mayer" cost function.

- The endpoints are the initial state $\boldsymbol{x}_{0} \equiv \boldsymbol{x}\left(t_{0}\right)$, the final state $\boldsymbol{x}_{f} \equiv \boldsymbol{x}\left(t_{f}\right)$, the initial time $t_{0}$ and the final time $t_{f}$.

- $F$ is the scalar running cost function. The arguments of $F$ are the instantaneous value of the state variable $\boldsymbol{x}(t)$, the instantaneous value of the control variable $\boldsymbol{u}(t)$ and time $t$.

- $\boldsymbol{f}$ is the $N_{x}$-dimensional "dynamics function," or more appropriately the right-hand-side of the dynamics equation. The arguments of $\boldsymbol{f}$ are exactly the same as the arguments of $F$.

- $\boldsymbol{e}$ is the $N_{e}$-dimensional endpoint constraint function. The arguments of $\boldsymbol{e}$ are exactly the same as that of $E$.

- $\boldsymbol{e}^{L}$ and $\boldsymbol{e}^{U}$ are the $N_{e}$-dimensional lower and upper bounds on the values of $\boldsymbol{e}$.

- $\boldsymbol{h}$ is the $N_{h}$-dimensional path constraint function. The arguments of $\boldsymbol{h}$ are exactly the same as that of $F$. 
- $\boldsymbol{h}^{L}$ and $\boldsymbol{h}^{U}$ are the $N_{h}$-dimensional lower and upper bounds on the values of $\boldsymbol{h}$.

As shown in [42], many key aspects of PS optimal control can be understood by considering a distilled optimal control problem given by,

$$
\begin{aligned}
& x \in \mathbb{R}, \quad u \in \mathbb{R}, \quad \tau \in[-1,1] \\
& (P)\left\{\begin{aligned}
\text { Minimize } & J[x(\cdot), u(\cdot)]= \\
\text { Subject to } & E(x(-1), x(1)) \\
\dot{x}(\tau) & =f(x(\tau), u(\tau)) \\
& e(x(-1), x(1))=0
\end{aligned}\right.
\end{aligned}
$$

The PS results generated for Problem $P$ easily generalize to Problem $G$, albeit with some tedious bookkeeping. In order to avoid the distractions of bookkeeping, we limit many of our subsequent discussions to Problem $P$ while noting that all of the results - with appropriate reindexing - apply to Problem $G$ as well. See also [1] for a more thorough review and survey of the key results.

In PS optimal control theory, the state trajectory $x(\cdot)$ is expressed - in principle - as a generalized Fourier expansion,

$$
x(\tau)=x^{\infty}(\tau):=\lim _{N \rightarrow \infty} x^{N}(\tau):=\lim _{N \rightarrow \infty} \sum_{m=0}^{N} a_{m} P_{m}(\tau)
$$

where, $a_{m} \in \mathbb{R}$ is an unknown "spectral" coefficient of an $m^{t h}$-order polynomial $P_{m}$. Note that (4) is an exact representation and not an approximation. The justification for (4) comes from the classic Stone-Weierstrass theorem[43] which guarantees a polynomial representation for $x(\cdot)$ under mild assumptions. For most aerospace applications, the "best" choices for $P_{m}$ are the Legendre and Chebyshev polynomials; hence, PS optimal control techniques resulting from these "big two" polynomials are known as the Legendre and Chebyshev PS methods respectively. A key principle in a generic PS method is that the spectral coefficients $a_{m}$ are computed indirectly by transforming (4) to an equivalent "pseudospectral" form[39, 44, 45],

$$
x^{N}(\tau):=\sum_{j=0}^{N} x^{N}\left(\tau_{j}\right) L_{j}(\tau)
$$

where, $\tau_{j}, j=0,1,2, \ldots, N$ are discrete points in time, known as nodes, and $L_{j}$ are Lagrange interpolating polynomials. That is, $L_{j}(\tau), j=0,1,2, \ldots, N$ are such that they satisfy the Kronecker relationship,

$$
L_{j}\left(\tau_{i}\right)=\delta_{i j}
$$

Equations (4) and (5) are known as the modal and nodal representations of $x(\cdot)$ respectively[39].

Let,

$$
\pi^{N}:=\left[\tau_{0}, \tau_{1}, \ldots, \tau_{N}\right]
$$

be an arbitrary grid (or "mesh") of points (see Fig. 4) such that

$$
-1 \leq \tau_{0}<\tau_{1}<\cdots<\tau_{N-1}<\tau_{N} \leq 1
$$

Note that (8) allows the extreme points of the grid, $\tau_{0}$ and $\tau_{N}$, to be at or near the endpoints. A nodal representation of the state trajectory over $\pi^{N}$ is given by,

$$
x^{N}(\tau):=\sum_{j=0}^{N} x_{j} L_{j}(\tau)
$$




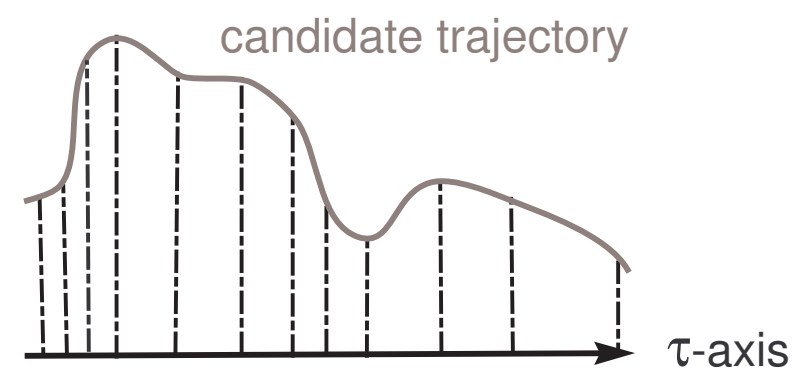

Figure 4. Illustration of an arbitrary grid in the generic pseudospectral method developed in [44] and [45].

Applying (6) to (9), it follows that $x_{i}=x^{N}\left(\tau_{i}\right), i=0, \ldots, N$. Differentiating (9) and evaluating $x^{N}(t)$ over the grid we get,

$$
\dot{x}^{N}\left(\tau_{i}\right)=\sum_{j=0}^{N} x_{j} \dot{L}_{j}\left(\tau_{i}\right)
$$

Define

$$
\boldsymbol{D}:=\left(\dot{L}_{j}\left(\tau_{i}\right)\right)_{0 \leq i, j \leq N}
$$

as the PS differentiation matrix, and let,

$$
X:=\left[x_{0}, x_{1}, \ldots, x_{N}\right]^{T}
$$

Then, using (11) and (12), (10) can be written as a matrix-vector product given by,

$$
\dot{X}=D X
$$

Note that in sharp contrast to Runge-Kutta methods, PS methods rely almost entirely on an accurate discretization of $\dot{x}$ rather than $f(x, u)$, the right-hand-side of the differential equation, (2).

A nodal representation of the control trajectory over $\pi^{N}$ is given by,

$$
u^{N}(\tau)=\sum_{j=0}^{N} u_{j} C_{j}(\tau)
$$

where, $C_{j}(\tau)$ is some interpolating function.

Remark 1 Note that the control trajectory $\tau \mapsto u^{N}(\tau)$ is not necessarily a polynomial. ${ }^{\text {a }}$ That is, $C_{j}(\tau)$ is any function that satisfies,

$$
C_{j}\left(\tau_{i}\right)=\delta_{i j}
$$

The non-polynomial form of $t \mapsto u^{N}(t)$ is exploited in [12, 13, 14, 15] for a strong proof of convergence; that is, a convergence proof that does not rely on unnecessary assumptions [46] that are typically not satisfied in many practical optimal control problems. Note as well that the Bellman pseudospectral method [47, 48] also capitalizes on the non-polynomial form of $t \mapsto u^{N}(t)$.

\footnotetext{
${ }^{a}$ Unfortunately, it is widely and erroneously reported in the optimal control literature that $C_{j}$ is a polynomial.
} 
Substituting (9) and (14) in $f(x, u)$, and evaluating $f$ over $\pi^{N}$ we get,

$$
\left.f\left(x^{N}(\tau), u^{N}(\tau)\right)\right|_{\pi^{N}}=\left[f\left(x_{0}, u_{0}\right), f\left(x_{1}, u_{1}\right), \ldots, f\left(x_{N}, u_{N}\right)\right]
$$

It is convenient to reuse $f$ as an overloaded function (see [39], page 8) defined by,

$$
f(X, U):=\left[f\left(x_{0}, u_{0}\right), f\left(x_{1}, u_{1}\right), \ldots, f\left(x_{N}, u_{N}\right)\right]^{T}
$$

where,

$$
U:=\left[u_{0}, u_{1}, \ldots, u_{N}\right]^{T}
$$

Setting $\dot{X}=f(X, U)$ and using (13), we define Problem $P^{N}$ according to,

$$
\begin{aligned}
& X \in \mathbb{R}^{N+1}, \quad U \in \mathbb{R}^{N+1} \\
& \left(P^{N}\right)\left\{\begin{aligned}
\text { Minimize } & J^{N}[X, U]:=E\left(x_{0}, x_{N}\right) \\
\text { Subject to } & \boldsymbol{D} X=f(X, U) \\
& e\left(x_{0}, x_{N}\right)=0
\end{aligned}\right.
\end{aligned}
$$

Comparing (3) and (16), it is clear that Problem $P^{N}$ is strikingly similar to Problem $P$. In fact, one may view the production of Problem $P^{N}$ as simple as the process of replacing the continuous operator $d / d t$ in Problem $P$ by the matrix $\boldsymbol{D}$.

Remark 2 In the derivation of Problem $P^{N}$, no assumption was made on the structure of the grid $\pi^{N}$; hence, (16) represents a discretization of Problem P for all PS methods [45].

Remark 3 Once $\pi^{N}$ is chosen for a given Problem P, the theoretical performance of a PS method depends only on the selection of the grid. The worst performance (i.e., rapid divergence) is characterized by a uniform distribution of nodes while the best performance (i.e., rapid convergence) requires node clustering at the end points [45].

Remark 3 is illustrated in Fig. 5. This figure was generated using the data and results from [45]. The parameter $\beta$ (see Fig. 5 a)) denotes a measure of clustering: from optimal clustering (i.e., when $\beta=0$ ) to a uniform node distribution (i.e., when $\beta=1$ ). It is apparent from Fig. 5 b) that optimal clustering is not required for convergence but is indeed critical for the rate of convergence. Hence, it is desirable to maintain node clustering for a fast mesh refinement.

Remark 4 If $\pi^{N}$ is a convergent grid, many of the properties of the continuous-time operator $d / d t$ are faithfully represented by $\boldsymbol{D}$. For instance, let $z$ be a function that is not identically equal to zero; then,

$$
\begin{gathered}
\text { if } \frac{d}{d t}(z)=0 \Rightarrow D Z=\mathbf{0} \\
\text { if } \frac{d^{2}}{d t^{2}}(z)=0 \Rightarrow D^{2} Z=\mathbf{0}
\end{gathered}
$$

From (17a), it follows that $D$ must be singular to accurately represent $d / d t$. Note also that $D$ must be square so that higher-order derivatives can be properly represented analogous to a repetitive application of $d / d t$ as implied in (17b). 


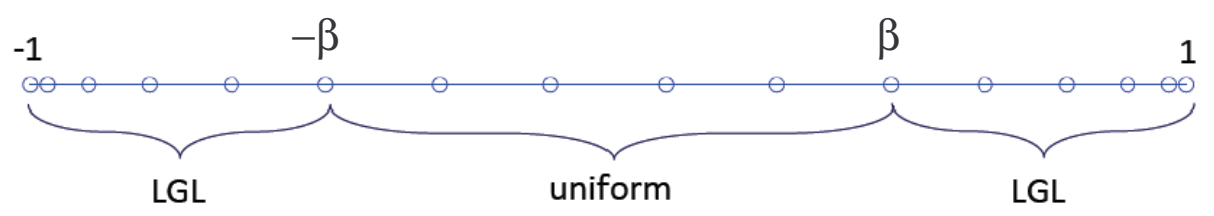

a)

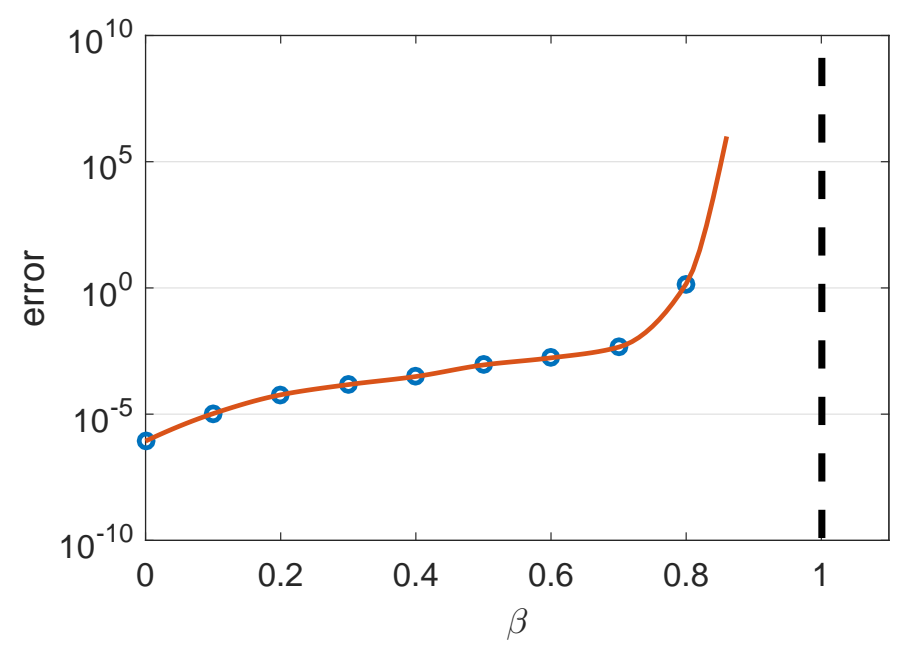

b)

Figure 5. Effect of node clustering on convergence of PS optimal control[45].

Remark 5 Node clustering is necessary for polynomial basis functions. It is possible to reduce node clustering through the use of non-polynomial basis functions [2, 49, 50]; however, the condition number of the resulting linear algebra still grows with an increase in $N$.

Problem $P^{N}$ is solved by the spectral algorithm[1, 11]. A key procedure for mesh refinement in the spectral algorithm for PS methods can be described as follows:

1. Select an initial sequence of integers $N_{0}<N_{1}<\cdots<N_{m}$ and generate a sequence of problems $P^{N_{k}}, k=0,1, \ldots, m$.

2. Solve Problem $N_{0}$ using the relaxed elastic programming concept described in [11]. Set $i=1$.

3. Solve Problem $N_{i}$ using the results from Problem $N_{i-1}$ by a joint application of PS convergence theory for optimal control[13, 14, 15] and the covector mapping principle[39].

4. Evaluate the (decay of the) spectral coefficients for convergence and stopping criteria[10, 11]. Exit if all stopping criteria are met; else, set $i=i+1$, and go to Step 3.

Many of the advancements in the spectral algorithm are implemented in DIDO ${ }^{\odot}[30,39]$, a stateof-the-art MATLAB ${ }^{\circledR}$ optimal control toolbox for solving optimal control problems. As noted earlier, the condition number of the linear matrix equations involved in the spectral algorithm grows as $N_{k}^{2}, k=0,1, \ldots, m$; hence, there is a loss in spectral accuracy and convergence rate 
on those problems that require a finer mesh. Among many, one of the current remedies for this problem is to use a pre-conditioner, $M$, such that the discretized differential equation in Problem $P^{N}$ can be written as,

$$
M D X=M f(X, U)
$$

The matrix $M$ must be chosen so that the condition number of the matrix equations resulting from the product $M D$ is lower than when $M$ is the identity matrix. If $M$ is not a diagonal matrix, then the sparsity of the right-hand-side of (18) decreases leading to an increase in the computational burden of the resulting linear algebra. Yet another remedy for managing the growth in the condition number is to use PS knots[8]. In fact, the full spectral algorithm incorporates an automatic determination of the number and location of knots based on a detection of rapid changes in the control function $[10,11]$. As noted in Section I, this approach is far from satisfactory. Although pre-conditioning and PS knots can be used jointly to mitigate the side-effects of both concepts, the resulting algorithm involves too many tuning parameters and inconsistent performance results. Clearly, there has been a lot of room for improvement in PS optimal control methods, particularly for those problems that require fast and accurate solutions over a fine mesh. As will be apparent shortly, the use of Birkhoff interpolating polynomials[51, 52] offers a new PS approach where solutions over thousands of points can be generated in a fast and stable manner.

\section{Birkhoff Interpolation Over an Arbitrary Grid}

In contrast to a Lagrange interpolant which is based on interpolating the values of a function, a Birkhoff interpolant is based on interpolating the values of a heterogenous mix of various orders of derivatives of a function[51]. That is, a Birkhoff interpolant generalizes Lagrange and Hermite interpolants[52]. For second-order boundary value problems (BVPs) with Dirichlet boundary conditions, a Birkhoff interpolation polynomial $\widetilde{x}^{N}(\tau)$ is given by[36],

$$
\widetilde{x}^{N}(\tau):=x\left(\tau_{0}\right) B_{0}(\tau)+\sum_{j=1}^{N-1} \ddot{x}\left(\tau_{j}\right) B_{j}(\tau)+x\left(\tau_{N}\right) B_{N}(\tau)
$$

where, $\left[\tau_{0}, \tau_{1}, \ldots, \tau_{N}\right]:=\pi^{N}$ is an arbitrary grid as before, and $B_{k}, k=0, \ldots, N$ are polynomials of order $N$ or less such that, if they exist, must satisfy the interpolation requirements,

$$
\widetilde{x}^{N}\left(\tau_{0}\right)=x\left(\tau_{0}\right), \quad \widetilde{x}^{N}\left(\tau_{N}\right)=x\left(\tau_{N}\right), \quad \ddot{\widetilde{x}}^{N}\left(\tau_{j}\right)=\ddot{x}\left(\tau_{j}\right), \quad j=1, \ldots, N-1
$$

Equation (20) generates the following conditions for the Birkhoff polynomials,

$$
\begin{aligned}
& B_{0}\left(\tau_{0}\right)=1 \quad B_{N}\left(\tau_{0}\right)=0 \quad B_{j}\left(\tau_{0}\right)=0, \quad j=1, \ldots, N-1 \\
& B_{0}\left(\tau_{N}\right)=0 \quad B_{N}\left(\tau_{N}\right)=1 \quad B_{j}\left(\tau_{N}\right)=0, \quad j=1, \ldots, N-1 \\
& \ddot{B}_{0}\left(\tau_{i}\right)=0 \quad \ddot{B}_{N}\left(\tau_{i}\right)=0 \quad \ddot{B}_{j}\left(\tau_{i}\right)=\delta_{i j} \quad i=1, \ldots, N-1 ; j=1, \ldots, N-1
\end{aligned}
$$

That is, for a second-order BVP, a Birkhoff interpolating polynomial may be based on a mix of the second derivatives of a function and its values at the boundary points. Because an optimal control problem also generates a BVP (through an application of the necessary conditions), it seems reasonable to simply rewrite (19) for first-order systems so that state-space representation may be used. This simple adaptation generates a proposal for a Birkhoff interpolant given by,

$$
\bar{x}^{N}(\tau):=x\left(\tau_{0}\right) B_{0}(\tau)+\sum_{i=1}^{N-1} \dot{x}\left(\tau_{i}\right) B_{i}(\tau)+x\left(\tau_{N}\right) B_{N}(\tau)
$$


where, $B_{k}, k=0, \ldots, N$ are polynomials (possibly different from those in (19)) that must satisfy,

$$
\bar{x}^{N}\left(\tau_{0}\right)=x\left(\tau_{0}\right), \quad \bar{x}^{N}\left(\tau_{N}\right)=x\left(\tau_{N}\right), \quad \dot{\bar{x}}^{N}\left(\tau_{i}\right)=\dot{x}\left(\tau_{i}\right)
$$

The conditions for (23) that are analogous to (21) are given by,

$$
\begin{aligned}
& B_{0}\left(\tau_{0}\right)=1 \quad B_{N}\left(\tau_{0}\right)=0 \quad B_{j}\left(\tau_{0}\right)=0, \quad j=1, \ldots, N-1 \\
& B_{0}\left(\tau_{N}\right)=0 \quad B_{N}\left(\tau_{N}\right)=1 \quad B_{j}\left(\tau_{N}\right)=0, \quad j=1, \ldots, N-1 \\
& \dot{B}_{0}\left(\tau_{i}\right)=0 \quad \dot{B}_{N}\left(\tau_{i}\right)=0 \quad \dot{B}_{j}\left(\tau_{i}\right)=\delta_{i j} \quad i=1, \ldots, N-1 ; j=1, \ldots, N-1
\end{aligned}
$$

The satisfaction of the conditions given by (24) has some well-known problems related to the existence and uniqueness of a solution[51, 52]; hence, we do not pursue this further. If the conditions on $B_{0}$ or $B_{N}$ are removed from (24), then it can be shown that a unique Birkhoff interpolant exists[53]. In doing the latter, a Birkhoff interpolant is given by,

$$
\text { Case (a): } \quad x^{N}(\tau):=x\left(\tau_{0}\right) B_{0}(\tau)+\sum_{j=1}^{N} \dot{x}\left(\tau_{j}\right) B_{j}(\tau)
$$

where, $B_{k}, k=0, \ldots, N$ must now only satisfy,

$$
x^{N}\left(\tau_{0}\right)=x\left(\tau_{0}\right), \quad \dot{x}^{N}\left(\tau_{j}\right)=\dot{x}\left(\tau_{j}\right), \quad j=1, \ldots, N
$$

Equation (26) generates fewer conditions than (24); these are given by,

$$
\begin{array}{lll}
B_{0}\left(\tau_{0}\right)=1, \quad B_{j}\left(\tau_{0}\right)=0, & j=1, \ldots, N \\
\dot{B}_{0}\left(\tau_{i}\right)=0, & \dot{B}_{j}\left(\tau_{i}\right)=\delta_{i j}, & i=1, \ldots, N
\end{array}
$$

In the same manner, an equivalent Birkhoff interpolant is given by,

\section{Case (b):}

$$
x^{N}(\tau):=\sum_{j=0}^{N-1} \dot{x}\left(\tau_{j}\right) B_{j}(\tau)+x\left(\tau_{N}\right) B_{N}(\tau)
$$

where, $B_{k}, k=0, \ldots, N$ are yet another set of polynomials that must satisfy,

$$
x^{N}\left(\tau_{N}\right)=x\left(\tau_{N}\right), \quad \dot{x}^{N}\left(\tau_{j}\right)=\dot{x}\left(\tau_{j}\right), \quad j=0, \ldots, N-1
$$

The conditions for $B_{k}, k=0, \ldots, N$ in (28) are given by,

$$
\begin{array}{llll}
B_{N}\left(\tau_{N}\right)=1, & B_{j}\left(\tau_{N}\right)=0, & j=0, \ldots, N-1 \\
\dot{B}_{N}\left(\tau_{i}\right)=0, & \dot{B}_{j}\left(\tau_{i}\right)=\delta_{i j}, & i=0, \ldots, N-1
\end{array}
$$

It is easy to show that a Birkhoff interpolant exists for both cases given by (25) and (28).

Remark 6 The Birkhoff interpolants given by (25) and (28) are over an arbitrary grid, $\pi^{N}$. Endpoint node clustering is necessary for convergence (see Fig. 5). Consequently, Birkhoff interpolants for Jacobi-Gauss-Lobatto, Jacobi-Gauss-Radau, and Jacobi-Gauss meshes follow from (25) and (28) by an appropriate choice of $\pi^{N}$. 
It will be apparent shortly that it is convenient to define two subsets of the grid $\pi^{N}$ given by,

$$
\begin{aligned}
\pi_{a}^{N} & :=\left[\tau_{1}, \ldots, \tau_{N}\right] \\
\pi_{b}^{N} & :=\left[\tau_{0}, \tau_{1}, \ldots, \tau_{N-1}\right]
\end{aligned}
$$

Thus, $\pi^{N}$ may be represented either by $\pi^{N}=\left[\tau_{0}, \pi_{a}^{N}\right]$ or $\pi^{N}=\left[\pi_{b}^{N}, \tau_{N}\right]$. Furthermore, we define the matrices,

$$
\begin{array}{rlrl}
\boldsymbol{D}_{a} & :=\left(\dot{L}_{j}\left(\tau_{i}\right)\right)_{1 \leq i, j \leq N} & \boldsymbol{B}_{a}:=\left(B_{j}\left(\tau_{i}\right)\right)_{1 \leq i, j \leq N} \\
\boldsymbol{D}_{b}:=\left(\dot{L}_{j}\left(\tau_{i}\right)\right)_{0 \leq i, j \leq N-1} & \boldsymbol{B}_{b}:=\left(B_{j}\left(\tau_{i}\right)\right)_{0 \leq i, j \leq N-1}
\end{array}
$$

where, $\boldsymbol{B}_{a}$ and $\boldsymbol{B}_{b}$ satisfy (27) and (30) respectively.

Theorem 1 (Wang-Samson-Zhao) Let $\omega \in\{a, b\}$. Then,

$$
\boldsymbol{D}_{\omega} \boldsymbol{B}_{\omega}=\boldsymbol{I}_{N}
$$

where, $\boldsymbol{I}_{N}$ is an $N \times N$ identity matrix.

Proof. The proof of this result for $\omega=a$ is implied in [36] by Theorems 3.2 and 4.1. For the purposes of completeness and clarity, we prove this theorem for an arbitrary grid by the same procedures used in [36]. Furthermore, because the matrix $\boldsymbol{B}_{b}$ is not presented in [36], we first prove this result for $\omega=b$. The proof for $\omega=a$ follows by a trivial extension.

For any $N^{t h}$-order polynomial $\phi$, we can write,

$$
\phi(\tau)=\sum_{k=0}^{N} \phi\left(\tau_{k}\right) L_{k}(\tau) \Rightarrow \dot{\phi}(\tau)=\sum_{k=0}^{N} \phi\left(\tau_{k}\right) \dot{L}_{k}(\tau)
$$

Setting $\phi(\tau)=B_{j}(\tau)$ in (33) and evaluating the resulting equation at $\tau_{i}$, we get,

$$
\dot{B}_{j}\left(\tau_{i}\right)=\sum_{k=0}^{N} B_{j}\left(\tau_{k}\right) \dot{L}_{k}\left(\tau_{i}\right)
$$

We can rewrite (34) for Case (b) evaluation as,

$$
\dot{B}_{j}\left(\tau_{i}\right)=\sum_{k=0}^{N-1} B_{j}\left(\tau_{k}\right) \dot{L}_{k}\left(\tau_{i}\right)+B_{j}\left(\tau_{N}\right) \dot{L}_{N}\left(\tau_{i}\right)
$$

Using (30) to evaluate (35) we get,

$$
\delta_{i j}=\sum_{k=0}^{N-1} B_{j}\left(\tau_{k}\right) \dot{L}_{k}\left(\tau_{i}\right), \quad 0 \leq i, j \leq N-1
$$

From (32), it is clear that (36) is the same as $\boldsymbol{D}_{\omega} \boldsymbol{B}_{\omega}=\boldsymbol{I}_{N}$ for $\omega=b$. It is also apparent that the proof for $\omega=a$ follows by a similar procedure. 


\section{A Family of Well-Conditioned PS Optimal Control Methods}

In computational optimal control, it is apparent that it is important to generate a reasonably accurate control trajectory $\left[t_{0}, t_{f}\right] \ni t \mapsto \boldsymbol{u}$. Furthermore, it is more important to generate an accurate value of the initial control than its final value because any error in $\boldsymbol{u}\left(t_{0}\right)$ will be amplified in terms of propagating the state trajectory through the dynamics $\dot{\boldsymbol{x}}=\boldsymbol{f}(\boldsymbol{x}, \boldsymbol{u}(t))$; see [30, 39] for details. This implies that the constraint at the initial point $\dot{\boldsymbol{x}}\left(t_{0}\right)=\boldsymbol{f}\left(\boldsymbol{x}\left(t_{0}\right), \boldsymbol{u}\left(t_{0}\right)\right)$ must be well-represented. In [36], initial value problems (IVPs) are solved using the matrix $\boldsymbol{B}_{a}$ and the differential equation is not imposed at the initial point. Because this approach is not satisfactory for optimal control applications, we need to modify existing results on Birkhoff interpolation for optimal control applications.

\section{A. Birkhoff PS Optimal Control Method Over an Arbitrary Grid Based on (25)}

In applying the results of the previous section to Problem $P$, we set,

$$
x^{N}(\tau):=x_{0} B_{0}(\tau)+\sum_{j=1}^{N} v_{j} B_{j}(\tau)
$$

where, $x_{0}$ and $v_{j}, j=1, \ldots, N$ are the unknown optimization variables over an arbitrary grid $\pi^{N}$, and $B_{k}, k=0,1, \ldots, N$ satisfy (27). Differentiating both sides of (37) we get,

$$
\dot{x}^{N}(\tau)=x_{0} \dot{B}_{0}(\tau)+\sum_{j=1}^{N} v_{j} \dot{B}_{j}(\tau)
$$

Substituting (38) in the dynamic constraint $\dot{x}^{N}(\tau)=f\left(x^{N}(\tau), u^{N}(\tau)\right)$, we get,

$$
x_{0} \dot{B}_{0}(\tau)+\sum_{j=1}^{N} v_{j} \dot{B}_{j}(\tau)=f\left(x^{N}(\tau), u^{N}(\tau)\right)
$$

Evaluating (39) over the grid $\pi^{N}=\left[\tau_{0}, \pi_{a}^{N}\right]$ we get,

$$
\begin{aligned}
I_{a} V_{a} & =f\left(x_{0}, u_{0}\right)-x_{0} \dot{B}_{0}\left(\tau_{0}\right) \\
V_{a} & =f\left(X_{a}, U_{a}\right)
\end{aligned}
$$

where, $I_{a}, V_{a}$ and $X_{a}$ are given by,

$$
\begin{aligned}
I_{a} & :=\left[\dot{B}_{1}\left(\tau_{0}\right), \ldots, \dot{B}_{N}\left(\tau_{0}\right)\right] \\
V_{a} & :=\left[v_{1}, \ldots, v_{N}\right]^{T} \\
X_{a} & :=\left[x^{N}\left(\tau_{1}\right), \ldots, x^{N}\left(\tau_{N}\right)\right]^{T}
\end{aligned}
$$

The vector $X_{a}$ can be evaluated using (37); this generates the constraint,

$$
X_{a}=x_{0} \boldsymbol{b}_{0}+\boldsymbol{B}_{a} V_{a}
$$

where,

$$
\boldsymbol{b}_{0}:=\left(\begin{array}{c}
B_{0}\left(\tau_{1}\right) \\
B_{0}\left(\tau_{2}\right) \\
\vdots \\
B_{0}\left(\tau_{N}\right)
\end{array}\right)
$$


Collecting all the relevant equations, we define

$$
\left(P_{a}^{N}\right)\left\{\begin{aligned}
X \in \mathbb{R}^{N+1}, \quad U \in \mathbb{R}^{N+1} & , \quad V_{a} \in \mathbb{R}^{N} \\
\text { Minimize } & J_{a}^{N}\left[X, U, V_{a}\right]:= \\
V_{a} & =f\left(x_{0}, x_{N}\right) \\
X_{a} & \left.=x_{0} \boldsymbol{b}_{0}+U_{a}\right) \\
I_{a} V_{a} & =f\left(x_{0}, u_{0}\right)-x_{0} \dot{B}_{0}\left(\tau_{0}\right) \\
e\left(x_{0}, x_{N}\right) & =0
\end{aligned}\right.
$$

Remark 7 The Birkhoff equality constraint $I_{a} V_{a}+x_{0} \dot{B}_{0}\left(\tau_{0}\right)-f\left(x_{0}, u_{0}\right)=0$ in Problem $P_{a}^{N}$ imposes the differential equation $\dot{x}(\tau)=f(x(\tau), u(\tau))$ at the initial point $\tau_{0}$. This Birkhoff constraint is equivalent to imposing the same differential equation via the Lagrange condition $\sum_{j=0}^{N} x_{j} \dot{L}_{j}\left(\tau_{0}\right)-f\left(x^{N}\left(\tau_{0}\right), u^{N}\left(\tau_{0}\right)\right)=0$. This equivalency is proved as Proposition 1.

Proposition 1 Let $x_{j}=x^{N}\left(\tau_{j}\right)$. At $\tau=\tau_{0}$, the Lagrange and Birkhoff interpolants satisfy the condition,

$$
\sum_{j=0}^{N} x_{j} \dot{L}_{j}\left(\tau_{0}\right)=x_{0} \dot{B}_{0}\left(\tau_{0}\right)+\sum_{j=1}^{N} v_{j} \dot{B}_{j}\left(\tau_{0}\right)
$$

Proof. The derivative $\dot{B}_{j}\left(\tau_{i}\right)$ can be obtained by an application of the PS differentiation matrix; hence, we have,

$$
\dot{B}_{j}\left(\tau_{i}\right)=\sum_{k=0}^{N} \dot{L}_{k}\left(\tau_{i}\right) B_{j}\left(\tau_{k}\right)
$$

Setting $i=j=0$ in (47) we get,

$$
\begin{aligned}
\dot{B}_{0}\left(\tau_{0}\right) & =\sum_{k=0}^{N} \dot{L}_{k}\left(\tau_{0}\right) B_{0}\left(\tau_{k}\right) \\
& =\dot{L}_{0}\left(\tau_{0}\right) B_{0}\left(\tau_{0}\right)+\sum_{k=1}^{N} \dot{L}_{k}\left(\tau_{0}\right) B_{0}\left(\tau_{k}\right) \\
& =\dot{L}_{0}\left(\tau_{0}\right)+\sum_{k=1}^{N} \dot{L}_{k}\left(\tau_{0}\right) B_{0}\left(\tau_{k}\right)
\end{aligned}
$$

where, the last equality in (48) follows from the fact that $B_{0}\left(\tau_{0}\right)=1$; see (27).

Similarly, setting $i=0$ in (47) we get,

$$
\begin{aligned}
\dot{B}_{j}\left(\tau_{0}\right) & =\sum_{k=0}^{N} \dot{L}_{k}\left(\tau_{0}\right) B_{j}\left(\tau_{k}\right) \\
& =\dot{L}_{0}\left(\tau_{0}\right) B_{j}\left(\tau_{0}\right)+\sum_{k=1}^{N} \dot{L}_{k}\left(\tau_{0}\right) B_{j}\left(\tau_{k}\right) \\
& =\sum_{k=1}^{N} \dot{L}_{k}\left(\tau_{0}\right) B_{j}\left(\tau_{k}\right)
\end{aligned}
$$


Evaluating the right-hand-side of (46) using (48) and (49) we have,

$$
x_{0} \dot{B}_{0}\left(\tau_{0}\right)+\sum_{j=1}^{N} v_{j} \dot{B}_{j}\left(\tau_{0}\right)=x_{0} \dot{L}_{0}\left(\tau_{0}\right)+\sum_{k=1}^{N} x_{0} \dot{L}_{k}\left(\tau_{0}\right) B_{0}\left(\tau_{k}\right)+\sum_{j=1}^{N} v_{j} \sum_{k=1}^{N} \dot{L}_{k}\left(\tau_{0}\right) B_{j}\left(\tau_{k}\right)
$$

Next, from (37), we have,

$$
x_{k}=x_{0} B_{0}\left(\tau_{k}\right)+\sum_{j=1}^{N} v_{j} B_{j}\left(\tau_{k}\right)
$$

Evaluating the left-hand-side of (46) using (51) we get,

$$
\begin{aligned}
\sum_{k=0}^{N} x_{k} \dot{L}_{k}\left(\tau_{0}\right) & =x_{0} \dot{L}_{0}\left(\tau_{0}\right)+\sum_{k=1}^{N} x_{k} \dot{L}_{k}\left(\tau_{0}\right) \\
& =x_{0} \dot{L}_{0}\left(\tau_{0}\right)+\sum_{k=1}^{N} x_{0} B_{0}\left(\tau_{k}\right) \dot{L}_{k}\left(\tau_{0}\right)+\sum_{k=1}^{N} \sum_{j=1}^{N} v_{j} B_{j}\left(\tau_{k}\right) \dot{L}_{k}\left(\tau_{0}\right)
\end{aligned}
$$

It is evident that the right-hand-sides of (50) and (52) are identical.

\section{B. Birkhoff PS Optimal Control Method Over an Arbitrary Grid Based on (28)}

Although many of the results are similar to those resulting from (37), we provide a brief development here to highlight the key differences between the two approaches. To this end, we set,

$$
x^{N}(\tau):=\sum_{j=0}^{N-1} v_{j} B_{j}(\tau)+x_{N} B_{N}(\tau)
$$

Substituting the (53) in the dynamic constraint we get,

$$
\sum_{j=0}^{N-1} v_{j} \dot{B}_{j}(\tau)+x_{N} \dot{B}_{N}(\tau)=f\left(x^{N}(\tau), u^{N}(\tau)\right)
$$

Evaluating (54) over the grid $\pi^{N}=\left[\pi_{b}^{N}, \tau_{N}\right]$ we get,

$$
\begin{aligned}
V_{b} & =f\left(X_{b}, U_{b}\right) \\
I_{b} V_{b} & =f\left(x_{N}, u_{N}\right)-x_{N} \dot{B}_{N}\left(\tau_{N}\right)
\end{aligned}
$$

where, $I_{b}, V_{b}$ and $X_{b}$ are given by,

$$
\begin{aligned}
I_{b} & :=\left[\dot{B}_{0}\left(\tau_{N}\right), \ldots, \dot{B}_{N-1}\left(\tau_{N}\right)\right] \\
V_{b} & :=\left[v_{0}, \ldots, v_{N-1}\right]^{T} \\
X_{b} & :=\left[x^{N}\left(\tau_{0}\right), \ldots, x^{N}\left(\tau_{N-1}\right)\right]^{T}
\end{aligned}
$$

Using (53) to evaluate $X_{b}$ we get,

$$
X_{b}=\boldsymbol{B}_{b} V_{b}+x_{N} \boldsymbol{b}_{N}
$$


where,

$$
\boldsymbol{b}_{N}:=\left(\begin{array}{c}
B_{N}\left(\tau_{0}\right) \\
B_{N}\left(\tau_{1}\right) \\
\vdots \\
B_{N}\left(\tau_{N-1}\right)
\end{array}\right)
$$

Thus, we now have a new discretization of Problem $P$ given by,

$$
\left(P_{b}^{N}\right)\left\{\begin{aligned}
& X \in \mathbb{R}^{N+1}, \quad U \in \mathbb{R}^{N+1}, \quad V_{b} \in \mathbb{R}^{N} \\
& \text { Minimize } J_{b}^{N}\left[X, U, V_{b}\right]:=E\left(x_{0}, x_{N}\right) \\
& \text { Subject to }=f\left(X_{b}, U_{b}\right) \\
& V_{b}=\boldsymbol{B}_{b} V_{b}+x_{N} \boldsymbol{b}_{N} \\
& I_{b} V_{b}=f\left(x_{N}, u_{N}\right)-x_{N} \dot{B}_{N}\left(\tau_{N}\right) \\
& e\left(x_{0}, x_{N}\right)=0
\end{aligned}\right.
$$

Remark 8 In Problem $P_{b}^{N}$, the differential constraint $\dot{x}(\tau)-f(x(\tau), u(\tau))=0$ at the initial point $\tau_{0}$ is imposed via the constraint $V_{b}-f\left(X_{b}, U_{b}\right)=0$. The Birkhoff equality constraint $I_{b} V_{b}+$ $x_{N} \dot{B}_{N}\left(\tau_{N}\right)-f\left(x_{N}, u_{N}\right)=0$ imposes the differential constraint at the final time. This Birkhoff condition is equivalent to imposing the same differential constraint via the Lagrange condition $\sum_{j=0}^{N} x_{j} \dot{L}_{j}\left(\tau_{N}\right)-f\left(x^{N}\left(\tau_{N}\right), u^{N}\left(\tau_{N}\right)\right)=0$. The proof of this equivalency is similar to Proposition 1 ; hence, it is omitted.

\section{Several Pre-Conditioned Lagrange PS Optimal Control Methods}

It is possible to develop several pre-conditioned Lagrange PS optimal control methods based on using Birkhoff matrices as pre-conditioners. We first consider the case where we partition the mesh $\pi^{N}$ as $\left[\tau_{0}, \pi_{a}^{N}\right]$.

\section{Left-Preconditioned Lagrange PS Method Based on $\left[\tau_{0}, \pi_{a}^{N}\right]$ Partitioning}

It is convenient to rewrite (9) as,

$$
x^{N}(\tau):=x_{0} L_{0}(\tau)+\sum_{i=1}^{N} x_{i} L_{i}(\tau)
$$

Differentiating (61) and substituting the result in the dynamic constraint $\dot{x}=f(x, u)$, we get,

$$
x_{0} \dot{L}_{0}(\tau)+\sum_{i=1}^{N} x_{i} \dot{L}_{i}(\tau)=f\left(x^{N}(\tau), u^{N}(\tau)\right)
$$

Evaluating (62) over the grid $\pi^{N}=\left[\tau_{0}, \pi_{a}^{N}\right]$ we get,

$$
\begin{aligned}
\sum_{i=0}^{N} x_{i} \dot{L}_{i}\left(\tau_{0}\right) & =f\left(x_{0}, u_{0}\right) \\
\boldsymbol{D}_{a} X_{a} & =f\left(X_{a}, U_{a}\right)-x_{0} \boldsymbol{l}_{0}
\end{aligned}
$$


where, $X_{a}$ and $U_{a}$ are defined in (42) and $l_{0}$ is defined by,

$$
\boldsymbol{l}_{0}:=\left(\begin{array}{c}
\dot{L}_{0}\left(\tau_{1}\right) \\
\dot{L}_{0}\left(\tau_{2}\right) \\
\vdots \\
\dot{L}_{0}\left(\tau_{N}\right)
\end{array}\right)
$$

Note that (63) is merely a partitioned version of the discrete differential constraint stipulated in Problem $P^{N}$ defined in (16). Pre-multiplying both sides of (63b) by $\boldsymbol{B}_{a}$ and using Theorem 1 we get,

$$
X_{a}=\boldsymbol{B}_{a} f\left(X_{a}, U_{a}\right)-x_{0} \boldsymbol{B}_{a} \boldsymbol{l}_{0}
$$

As a result of (65), Problem $P^{N}$ transforms according to,

$$
\begin{aligned}
& X \in \mathbb{R}^{N+1}, \quad U \in \mathbb{R}^{N+1} \\
& \left(\text { Left- } P_{a}^{N}\right)\left\{\begin{array}{rlrl}
\text { Minimize } & J^{N}[X, U]: & =E\left(x_{0}, x_{N}\right) \\
\text { Subject to } & \sum_{i=0}^{N} x_{i} \dot{L}_{i}\left(\tau_{0}\right) & =f\left(x_{0}, u_{0}\right) \\
X_{a} & =\boldsymbol{B}_{a} f\left(X_{a}, U_{a}\right)-x_{0} \boldsymbol{B}_{a} \boldsymbol{l}_{0} \\
e\left(x_{0}, x_{N}\right) & =0
\end{array}\right.
\end{aligned}
$$

\section{Right-Preconditioned Lagrange PS Method Based on $\left[\tau_{0}, \pi_{a}^{N}\right]$ Partitioning}

Instead of pre-multiplying equations to achieve better conditioning, we can generate an alternative method by post-multiplying only the left-hand-side of (63b) by $B_{a}$. To clarify this point, we substitute (43) in (63b); this results in,

$$
V_{a}+x_{0} \boldsymbol{D}_{a} \boldsymbol{b}_{0}=f\left(X_{a}, U_{a}\right)-x_{0} \boldsymbol{l}_{0}
$$

Comparing (67) with (41), it is evident that we need to prove that $x_{0} \boldsymbol{D}_{a} \boldsymbol{b}_{0}=-x_{0} \boldsymbol{l}_{0}$. This result is proved as Proposition 2.

Proposition $2 D_{a} b_{0}+l_{0}=0$

Proof. From (47), we have,

$$
\dot{B}_{j}\left(\tau_{i}\right)=\dot{L}_{0}\left(\tau_{i}\right) B_{j}\left(\tau_{0}\right)+\sum_{k=1}^{N} \dot{L}_{k}\left(\tau_{i}\right) B_{j}\left(\tau_{k}\right)
$$

Evaluating (68) for $j=0$ and $i=1, \ldots N$, we get,

$$
\left(\begin{array}{c}
\dot{B}_{0}\left(\tau_{1}\right) \\
\vdots \\
\dot{B}_{0}\left(\tau_{N}\right)
\end{array}\right)=\left(\begin{array}{c}
\dot{L}_{0}\left(\tau_{1}\right) \\
\vdots \\
\dot{L}_{0}\left(\tau_{N}\right)
\end{array}\right) B_{0}\left(\tau_{0}\right)+\boldsymbol{D}_{a} \boldsymbol{b}_{0}
$$

The left-hand-side of (69) is zero from (27); hence, the proposition follows from (64) and the fact that $B_{0}\left(\tau_{0}\right)=1$ by definition. 
As a result of Proposition 2, (67) simplifies to $V_{a}=f\left(X_{a}, U_{a}\right)$. Using Proposition 1 to replace (63a), Problem $P^{N}$ now transforms to a "right-preconditioned" method given by,

$$
\left(\text { right- } P_{a}^{N}\right)\left\{\begin{aligned}
X \in \mathbb{R}^{N+1}, \quad U \in \mathbb{R}^{N+1}, & V_{a} \in \mathbb{R}^{N} \\
\text { Minimize } & J_{a}^{N}\left[X, U, V_{a}\right]:= \\
\text { Subject to } & E\left(x_{0}, x_{N}\right) \\
I_{a} V_{a}= & f\left(x_{0}, u_{0}\right)-x_{0} \dot{B}_{0}\left(\tau_{0}\right) \\
V_{a}= & f\left(X_{a}, U_{a}\right) \\
X_{a}= & x_{0} \boldsymbol{b}_{0}+\boldsymbol{B}_{a} V_{a} \\
e\left(x_{0}, x_{N}\right) & =0
\end{aligned}\right.
$$

It is apparent that (70) is identical to Problem $P_{a}^{N}$ defined by (45). In other words, the Birkhoff-PS method (for Case (a)) may be viewed as a right-pre-conditioned Lagrange-PS method.

Proposition 3 If the variable $V_{a}$ is eliminated from Problem right- $P_{a}^{N}$, it reduces to Problem left$P_{a}^{N}$.

Proof. Comparing (66) with (70), it follows that this proposition is proved if we can show that:

(1) $\boldsymbol{B}_{a} f\left(X_{a}, U_{a}\right)-x_{0} \boldsymbol{B}_{a} \boldsymbol{l}_{0}=x_{0} \boldsymbol{b}_{0}+\boldsymbol{B}_{a} V_{a}$, and

(2) the equation $\sum_{i=0}^{N} x_{i} \dot{L}_{i}\left(\tau_{0}\right)=f\left(x_{0}, u_{0}\right)$ is equivalent to $I_{a} V_{a}=f\left(x_{0}, u_{0}\right)-x_{0} \dot{B}_{0}\left(\tau_{0}\right)$

Hence, we prove this proposition in two parts.

Part (1):

Substituting (41) in (43), we get,

$$
\begin{aligned}
x_{0} \boldsymbol{b}_{0}+\boldsymbol{B}_{a} V_{a} & =x_{0} \boldsymbol{b}_{0}+\boldsymbol{B}_{a} f\left(X_{a}, U_{a}\right) \\
& =-x_{0} \boldsymbol{B}_{a} \boldsymbol{l}_{0}+\boldsymbol{B}_{a} f\left(X_{a}, U_{a}\right)
\end{aligned}
$$

where, the last equality in (71) follows from Proposition (2) and Theorem 1.

Part (2):

The proof of this part is a direct consequence of Proposition 1.

Remark 9 It is clear that two additional left and right pre-conditioned Lagrange-PS methods can be similarly obtained by using the (Case (b)) partitioning $\pi^{N}=\left[\pi_{b}^{N}, \tau_{N}\right]$. For the purposes of brevity, these cases are not derived.

Remark 10 For Case (a), it is straightforward to show that $B_{0}(\tau)=1$ for all $\tau \in\left[\tau_{0}, \tau_{N}\right]$. Consequently, $\boldsymbol{b}_{0}$ defined in (44) is a vector of ones and $\dot{B}_{0}\left(\tau_{0}\right)=0$. Similarly, for Case (b), $B_{N}(\tau)=1$ for all $\tau \in\left[\tau_{0}, \tau_{N}\right]$; hence, $\boldsymbol{b}_{N}$ defined in (59) is a vector of ones and $\dot{B}_{N}\left(\tau_{N}\right)=0$.

Remark 11 All PS methods proposed in this section are for an arbitrary grid. It is possible to generate a very large sub-class of each of these methods by choosing slightly different and wellknown grid points. Popular examples of such grid points are Chebyshev-Gauss-Lobatto (CGL), Legendre-Gauss-Lobatto (LGL), Chebyshev-Gauss-Radau (CGR), Legendre-Gauss-Radau (LGR), Chebyshev-Gauss (CG) and Legendre-Gauss (LG). See [1, 42, 45] for further details on advantages and pitfalls associated with the choice of these mesh points as they apply to computational optimal control problems.

Because it is possible to generate a very large family of PS methods based on the results of the preceding subsections, we limit the scope of the present paper to illustrating only a small set of these methods. A more extensive discussion of other options and numerical results are discussed in [54]. Nonetheless, we note that a selection of the proper grid for optimal control problems is largely based on whether or not the horizon is finite or infinite[39]. 


\section{A Numerical Demonstration of the Well-Conditioning of Birkhoff PS Discretizations}

A quick examination of (70) shows that the linear equation given by (43) is the only equation in a Birkhoff PS method (for Case (a)) that is independent of the problem data functions $E, e$ and $f$. Consequently, if the original continuous-time problem is well-conditioned, the conditioning of the discretized problem is driven by the condition numbers of the linear equation given by (43) for Case (a), and by (58) for Case (b). Because all conclusions drawn with respect to the condition number of Case (a) are identical to that of Case (b), we focus the entirety of our discussions in this section to that of Case (a) only.

A very powerful statement on the condition number of the Birkhoff PS discretization can be drawn for the special case of a known value of the initial condition, $x_{0}$. In this case, we can re-write (43) as,

$$
\boldsymbol{B}_{a} V_{a}-X_{a}=-x_{0} \boldsymbol{b}_{0}
$$

where, the right-hand-side of (72) is a known quantity. Hence, the condition number of a Birkhoff PS discretization is driven by the condition number of the $(N \times 2 N)$-matrix $C_{N}^{\text {Birk }}$ defined by,

$$
\boldsymbol{C}_{N}^{\text {Birk }}:=\left[\boldsymbol{B}_{a},-\boldsymbol{I}_{N}\right]
$$

where $\boldsymbol{I}_{N}$ is the $N \times N$ identity matrix. As evident from Fig. 6, the condition number of $\boldsymbol{C}_{N}^{\text {Birk }}$

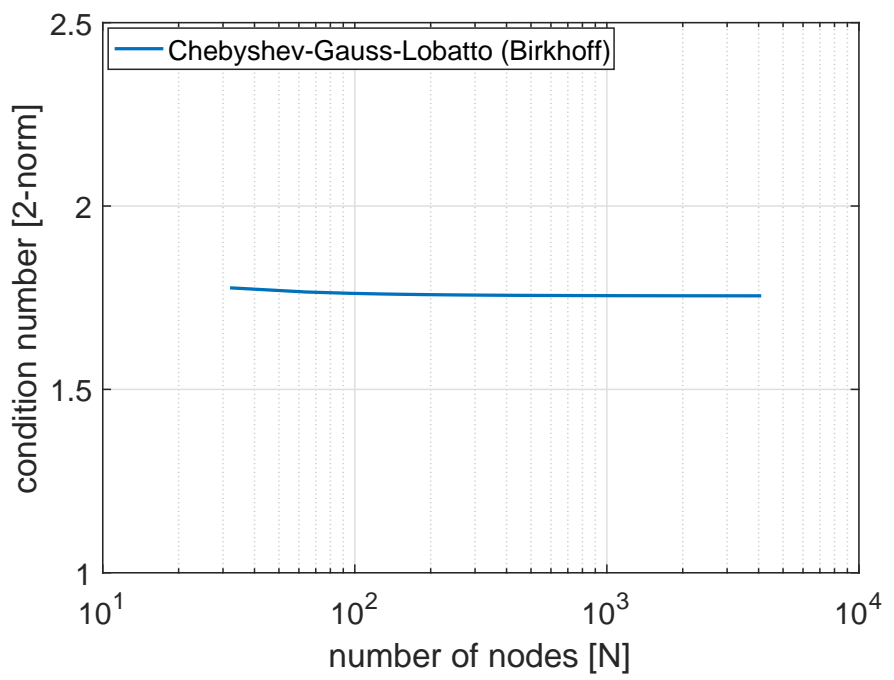

Figure 6. Demonstrating $\mathcal{O}(1)$ variation in the condition number of the matrix $C_{N}^{\text {Birk }}$ defined in (73) with evaluations over $N$ CGL nodes.

is remarkably flat with respect to $N$; i.e., of $\mathcal{O}(1)$. The condition number of a corresponding Lagrange PS discretization may be obtained by multiplying both sides of (72) by $\boldsymbol{D}_{a}$ and invoking Theorem 1. This results in

$$
\boldsymbol{D}_{a} X_{a}-V_{a}=x_{0} \boldsymbol{D}_{a} \boldsymbol{b}_{0}
$$

with the right-hand-side of (74) considered to be a known quantity. Hence, the condition number of a Lagrange PS discretization is driven by the condition number of the $(N \times 2 N)$-matrix,

$$
\boldsymbol{C}_{N}^{\mathrm{Lagr}}:=\left[\boldsymbol{D}_{a},-\boldsymbol{I}_{N}\right]
$$




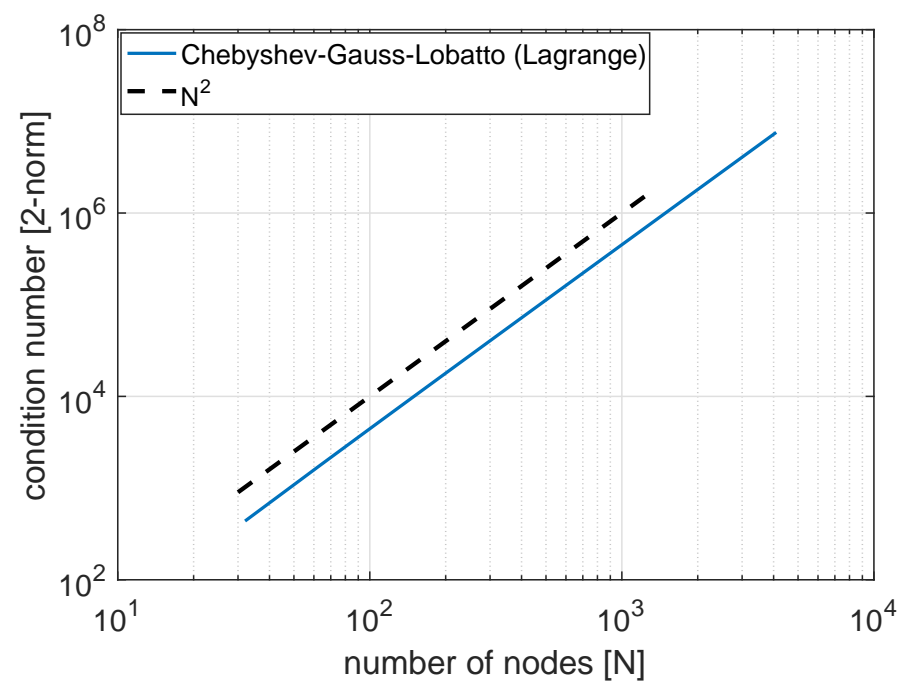

Figure 7. $\mathcal{O}\left(N^{2}\right)$-growth in the condition number of the matrix $C_{N}^{\text {Lagr }}$ defined in (75) with evaluations over $N$ CGL nodes.

It is clear from Fig. 7 that the condition number of $C_{N}^{\mathrm{Lagr}}$ is $\mathcal{O}\left(N^{2}\right)$ and consistent with the plot shown in Fig. 2.

In a generic optimal control problem, $x_{0}$ is an optimization variable. In this general case, the right-hand-side of (72) is not known; hence, we need to examine the condition number of the $N \times(2 N+1)$-matrix,

$$
\boldsymbol{A}_{N}^{\text {Birk }}:=\left[\boldsymbol{B}_{a},-\boldsymbol{I}_{N}, \boldsymbol{b}_{0}\right]
$$

It is apparent from Fig. 8 that the condition number of $\boldsymbol{A}_{N}^{\text {Birk }}$ varies only as $\mathcal{O}(\sqrt{N})$.

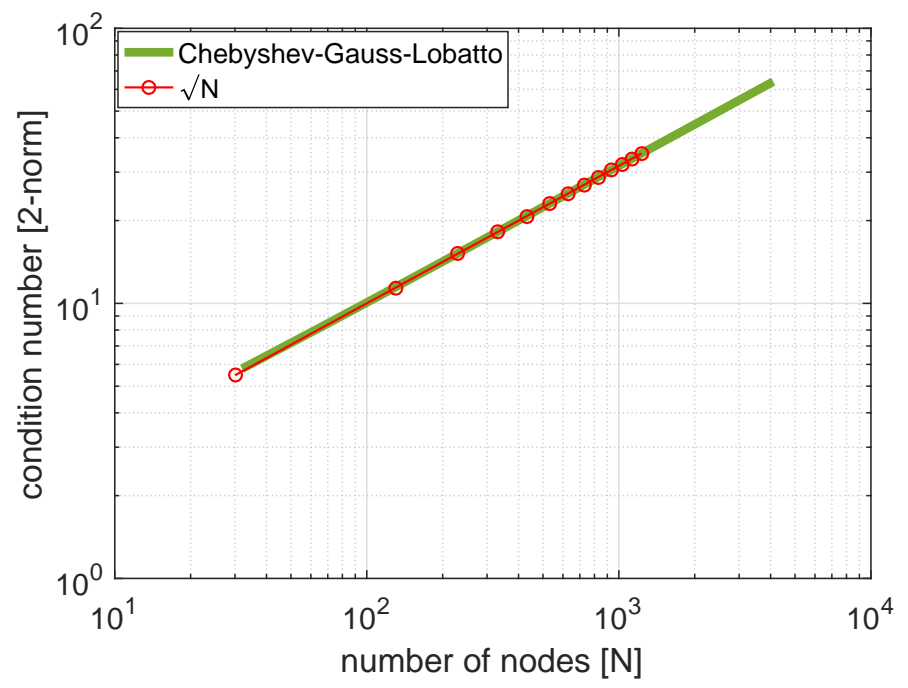

Figure 8. Demonstrating $\mathcal{O}(\sqrt{N})$-growth in the condition number of the matrix $\boldsymbol{A}_{N}^{\text {Birk }}$ defined in (76) with evaluations over $N$ CGL nodes.

The condition numbers for other choices of grid selections (see Remark 11) have similar behavior; see, for example, Fig. 2. In this paper we largely focus on Lobatto-based grid selections 
because they are the correct choice for solving generic finite-horizon optimal control problems (see for example, Ref. [1], Section 4.4.4 in Ross[39], and Ref. [42]). Radau-based mesh points - first introduced in [55] and [56] for optimal control applications - are more appropriate for infinite-horizon optimal control problems because the non-unit weight function associated with the Legendre-Radau-grid vanishes at the +1 (or -1 ) point $[1,56]$. Consequently, a Legendre-Radau grid is mathematically justified only for some limited choices of boundary conditions in a finitehorizon optimal control problem[1, 42]. Using a Radau mesh for finite-horizon optimal control applications with arbitrary boundary conditions produces well-known convergence issues and control-chatter problems [1, 39, 42]. Pure Gauss points are even more limiting with regards to their suitability for solving generic optimal control problems[1, 42].

\section{An Illustrative Example}

There are many emerging problems in aerospace trajectory optimization that require a fine $\operatorname{mesh}[31,32,33,57]$. A detailed discussion of these problems is beyond the scope of this paper; hence, we use a proxy low-thrust orbital maneuvering problem $[47,57]$ as a prototypical challenge to illustrate the numerics of the new PS methods developed in the preceding sections. That is, we choose this problem for illustrative purposes only and not necessarily to address the myriad of issues that are associated with the specifics of low-thrust orbital maneuvering. To this end, consider a minimum-time circle-to-circle continuous thrust orbit transfer problem (see Fig. 9) given by,

$$
\left(O _ { \text { Xfer } ) } \left\{\begin{array}{rl}
\boldsymbol{x}=\left(r, \theta, v_{r}, v_{t}\right) \in \mathbb{R}^{4}, & \boldsymbol{u}=\alpha \in \mathbb{R} \\
\text { Subject to } \quad J\left[\boldsymbol{x}(\cdot), \boldsymbol{u}(\cdot), t_{f}\right] & =t_{f} \\
\dot{r} & =v_{r} \\
\dot{\theta} & =\frac{v_{t}}{r} \\
\dot{v}_{r} & =\frac{v_{t}^{2}}{r}-\frac{\mu}{r^{2}}+A \sin \alpha \\
\dot{v}_{t} & =-\frac{v_{r} v_{t}}{r}+A \cos \alpha \\
t_{0} & =0 \\
\left(r_{0}, \theta_{0}, v_{r_{0}}, v_{t_{0}}\right) & =\left(r^{0}, 0,0, \sqrt{\mu / r^{0}}\right) \\
\left(r_{f}, v_{r_{f}}, v_{t_{f}}\right) & =\left(r^{f}, 0, \sqrt{\mu / r^{f}}\right)
\end{array}\right.\right.
$$

where, $r$ is the radial position of the spacecraft, $\theta$ is the true anomaly, $v_{r}$ and $v_{t}$ are the radial and transverse components of the spacecraft velocity, $\alpha$ is the thrust steering angle, $\mu$ is the gravitational parameter and $A$ is the magnitude of the constant thrust acceleration. What makes Problem $O_{\text {Xfer }}$ challenging is that if $A$ is low and $\left(r^{f} / r^{0}\right)$ is high, then the number of orbital revolutions to attain the final orbit is high; hence, from an elementary application of the Nyquist-Shannon sampling theorem, it is apparent that the number of nodes must be high. In principle, solving $\boldsymbol{a}$ problem with a large number of nodes is not a significant technological hurdle[31, 39]. As an elementary illustration of the technology requirements, we note that a million variables only requires $8 \mathrm{MB}$ of computer memory. What makes a high-node problem hard to solve is science and 


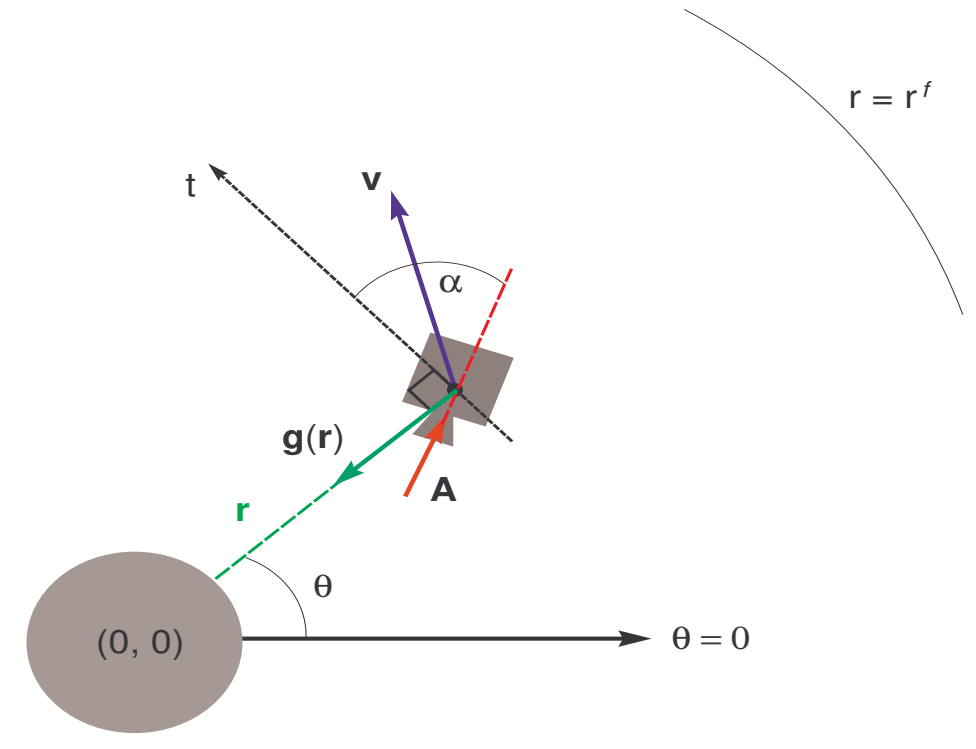

Figure 9. Schematic for Problem $O_{\text {Xfer }}$.

not technology; that is, the problem is the high condition number of the differentiation matrix associated with a PS method. For example, from Fig. 2, the condition number of a PS differentiation matrix for $N=1000$ is about $10^{6}$; i.e., $\mathcal{O}\left(N^{2}\right)$. Because it is equivalent to a right-preconditioned Lagrange PS method (see (70)), a Birkhoff PS method mitigates the problem of high condition numbers. For example, for $N=1000$, we expect the condition number to be about 30; see Fig. 8. To illustrate these features, we solve Problem $O_{\text {Xfer }}$ using the following canonical units:

$$
\begin{aligned}
\text { Distance Unit } & =r^{0} \\
\text { Speed Unit } & =\sqrt{\mu / r^{0}} \\
\text { Time Unit } & =\sqrt{\left(r^{0}\right)^{3} / \mu} \\
\text { Acceleration Unit } & =\mu /\left(r^{0}\right)^{2}
\end{aligned}
$$

The boundary conditions for a representative LEO-to-GEO orbit transfer problem (in canonical units) is given by,

$$
\begin{aligned}
\left(r_{0}, \theta_{0}, v_{r_{0}}, v_{t_{0}}\right) & =(1,0,0,1) \\
\left(r_{f}, v_{r_{f}}, v_{t_{f}}\right) & =(6,0, \sqrt{1 / 6})
\end{aligned}
$$

A low-thrust solution $\left(A=5 \times 10^{-4}\right)$ resulting from an application of Birkhoff PS method is shown in Fig. 10. This solution was obtained using a CGL mesh and the right preconditioning method defined by (70). Results for other cases and problems including solutions from the vast variety of other possible Birkhoff PS methods outlined in Remark 11 are discussed in [54]. In order to limit the scope of this section, we only discuss this particular case.

The time to accomplish the maneuver shown in Fig. 10 was approximately 1187 time units; this translates to approximately 13 days of continuous low-thrust maneuvering. The number of CGL points was $N=1024$. In other words, $r(t)$ shown in Fig. 10 is given by a polynomial of over a thousand degree! The "discrete" steering angle at 1024 CGL points is shown in Fig. 11. When this steering angle is interpolated through the CGL mesh, we get the continuous function $t \mapsto \alpha$ 


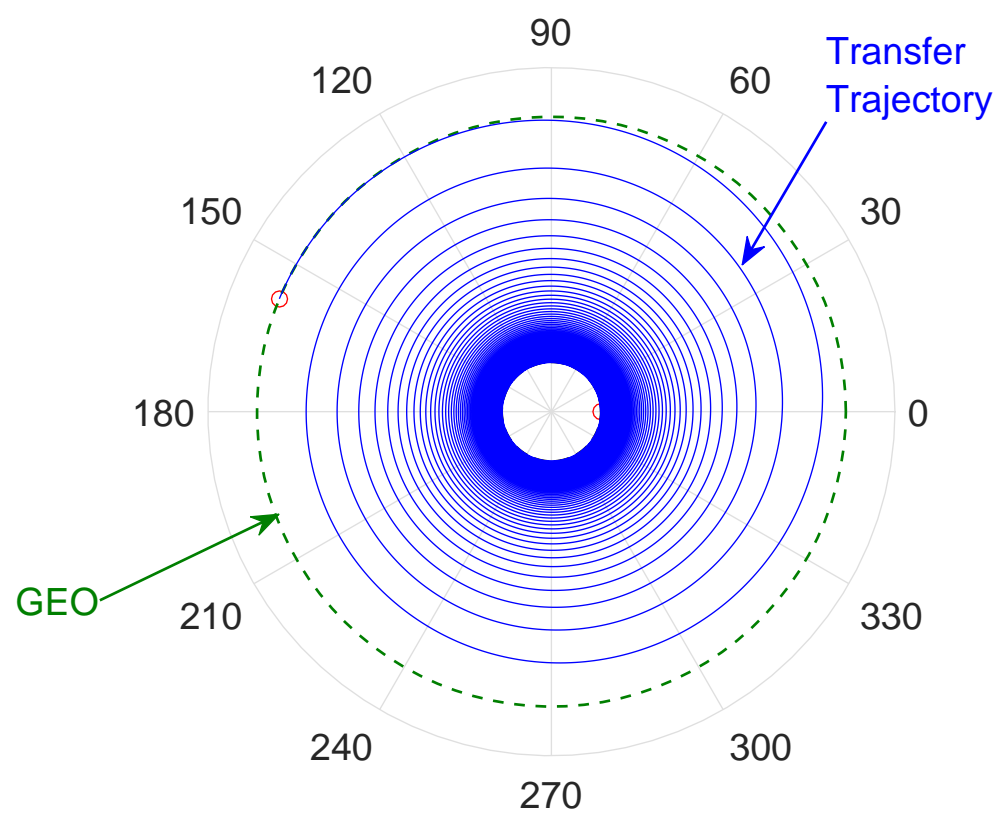

Figure 10. A Birkhoff PS solution for Problem $O_{\text {Xfer }}$ and boundary conditions given by (78).

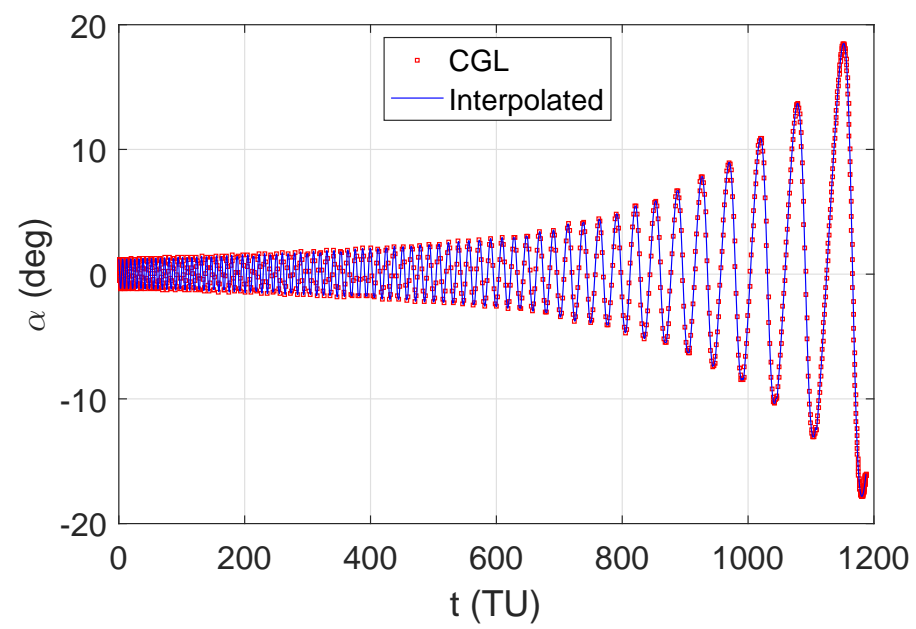

Figure 11. A CGL-interpolated PS control solution $t \mapsto \alpha(t)$ corresponding to the plot shown in Fig. 10.

indicated by the solid line in Fig. 11. Using the interpolated function $t \mapsto \alpha$, the dynamical equations can be easily integrated using ode45 (from MATLAB) to generate a propagated trajectory. This propagated trajectory is included in Fig. 10; however, this plot is not noticeable in this figure because the differences are too small to be visually apparent. Consequently, we provide an error plot in Fig. 12 as a matter of completeness.

\section{Conclusions}

State trajectories in aerospace dynamical systems are absolutely continuous; hence, by an elementary application of the Stone-Weierstrass theorem, they can be expressed to any arbitrary 


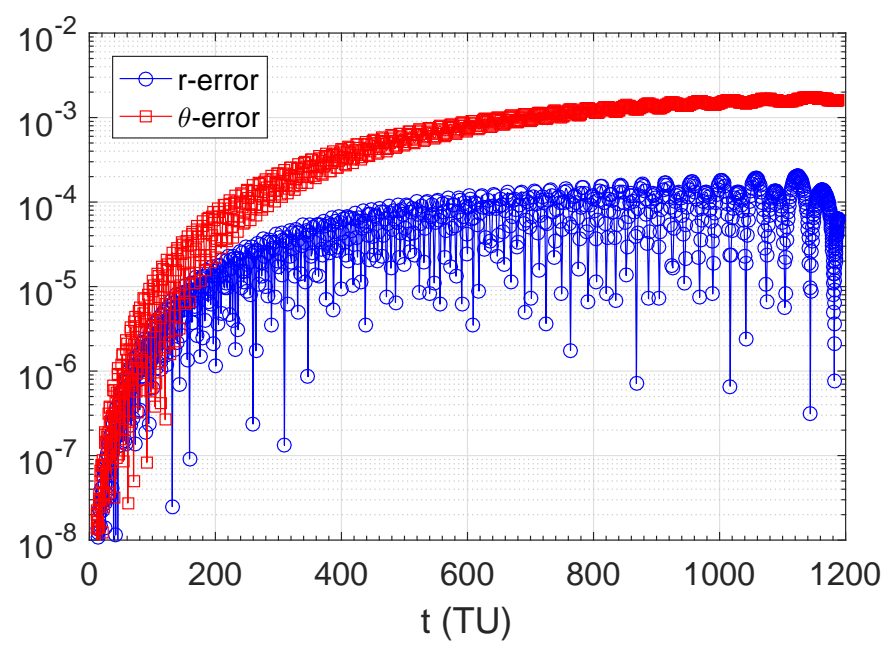

Figure 12. Error plots as measured by the difference between a Birkhoff PS solution and a RungeKutta propagation through the CGL-interpolated solution shown in Fig. 11.

precision in terms of some finite-order polynomial. In using the big two polynomials, namely Legendre and Chebyshev, pseudospectral (PS) optimal control theory has found numerous applications that include include flight implementations in aerospace engineering. In many practical PS optimal control applications, very accurate solutions can be generated using polynomials of order $N \leq 100$, thanks to the spectral (i.e., near exponential) rate of convergence. Emerging applications have sought to generate solutions in excess of $N>1000$. The challenge with such high orders is the high condition number $\left(\sim \mathcal{O}\left(N^{2}\right)\right)$ of the associated differentiation matrix. This is largely a science problem rather than a technological one. That is, a computer's processing capability is not a limiting factor because $8 \mathrm{MB}$ of memory for a million variables is no longer a technological hurdle. New advancements in the science associated with Birkhoff interpolation provide a dramatic drop in the condition number: from $\mathcal{O}\left(N^{2}\right)$ to $\mathcal{O}(\sqrt{N})$ or even $\mathcal{O}(1)$ in some cases. In advancing these new ideas, the problem with condition numbers is now relegated to specific applications and not to PS optimal control techniques. Nonetheless, a significant amount of new research on Birkhoff PS methods remains to be done.

\section{Acknowledgment}

We take this opportunity to thank the anonymous reviewers for providing many detailed comments. Their comments greatly improved the quality of this paper.

\section{References}

[1] I. M. Ross and M. Karpenko, "A Review of Pseudospectral Optimal Control: From Theory to Flight," Annual Reviews in Control, Vol.36, No.2, pp.182-197, 2012.

[2] J. Boyd, Chebyshev and Fourier Spectral Methods, Dover Publications, Inc., Minola, New York, 2001.

[3] F. Fahroo and I. M. Ross, "Direct Trajectory Optimization by a Chebyshev Pseudospectral Method,' Journal of Guidance, Control and Dynamics, Vol. 25, No. 1, 2002, pp.160-166. 
[4] Q. Gong, I. M. Ross and F. Fahroo, "Costate Computation by a Chebyshev Pseudospectral Method," Journal of Guidance, Control and Dynamics, Vol. 33, No. 2, pp. 623-628, 2010.

[5] L. N. Trefethen, Approximation Theory and Approximation Practice, SIAM, Philadelphia, PA, 2013.

[6] J. Shen, T. Tang and L.-L. Wang, Spectral Methods: Algorithms, Analysis and Applications, Springer, Heidelberg, 2011.

[7] F. Fahroo and I. M. Ross, "A Spectral Patching Method for Direct Trajectory Optimization," Journal of the Astronautical Sciences, Vol. 48, No. 2/3, April-September 2000, pp. 269-286.

[8] I. M. Ross and F. Fahroo, "Pseudospectral Knotting Methods for Solving Optimal Control Problems," Journal of Guidance, Control and Dynamics, Vol. 27, No. 3, pp. 397-405, 2004.

[9] I. M. Ross and F. Fahroo, "Discrete Verification of Necessary Conditions for Switched Nonlinear Optimal Control Systems," Proceedings of the American Control Conference, June 2004, Boston, MA.

[10] Q. Gong and I. M. Ross, “Autonomous Pseudospectral Knotting Methods for Space Mission Optimization," Advances in the Astronatuical Sciences, Vol. 124, 2006, AAS 06-151, pp. 779-794.

[11] Q. Gong, F. Fahroo and I. M. Ross, "Spectral Algorithm for Pseudospectral Methods in Optimal Control," Journal of Guidance, Control, and Dynamics, vol. 31 no. 3, pp. 460-471, 2008.

[12] Q. Gong, W. Kang and I. M. Ross, "A Pseudospectral Method for the Optimal Control of Constrained Feedback Linearizable Systems," IEEE Transactions on Automatic Control, Vol. 51, No. 7, July 2006, pp. 1115-1129.

[13] W. Kang, Q. Gong and I. M. Ross, "On the Convergence of Nonlinear Optimal Control Using Pseudospectral Methods for Feedback Linearizable Systems," International Journal of Robust and Nonlinear Control, Vol. 17, pp. 1251-1277, 2007.

[14] W. Kang, I. M. Ross and Q. Gong, "Pseudospectral Optimal Control and its Convergence Theorems," Analysis and Design of Nonlinear Control Systems, Springer-Verlag, Berlin Heidelberg, 2008, pp. 109-126.

[15] W. Kang, "Rate of Convergence for a Legendre Pseudospectral Optimal Control of Feedback Linearizable Systems," Journal of Control Theory and Applications, Vol. 8, No. 4, pp. 391405, 2010.

[16] A. Herman and B. A. Conway, "Direct Optimization Using Collocation Based on High-Order Gauss-Lobatto Quadrature Rules," Journal of Guidance Control and Dynamics, Vol.39, No.3, 1996, pp.592-599.

[17] E. Hairer, S. P. Nørsett, G. Wanner, Solving Ordinary Differential Equations I: Nonstiff Problems, Springer-Verlag Berlin Heidelberg, 1993. 
[18] B. A. Conway, "A Survey of Methods Available for the Numerical Optimization of Continuous Dynamic Systems," Journal of Optimization Theory and Applications, Vol. 152, 2012, pp. 271-306.

[19] M. Karpenko, C. J. Dennehy, H. C. Marsh and Q. Gong, "Minimum Power Slews and the James Webb Space Telescope," 27th AAS/AIAA Space Flight Mechanics Meeting, February 5-9, San Antonio, TX. Paper number: AAS-17-285.

[20] M. Karpenko, I. M. Ross, E. Stoneking, K. Lebsock and C. J. Dennehy, "A Micro-Slew Concept for Precision Pointing of the Kepler Spacecraft," AAS/AIAA Astrodynamics Specialist Conference, August 9-13, 2015, Vail, CO. Paper number: AAS-15-628.

[21] R. E. Stevens and W. Wiesel, "Large Time Scale Optimal Control of an Electrodynamic Tether Satellite," Journal of Guidance, Control and Dynamics, Vol. 32, No. 6, pp.1716-1727, 2008.

[22] Q. Gong, W. Kang, N. Bedrossian, F. Fahroo, P. Sekhavat and K. Bollino, "Pseudospectral Optimal Control for Military and Industrial Applications," 46th IEEE Conference on Decision and Control, 2007, pp. 4128-4142.

[23] N. Bedrossian, S. Bhatt, W. Kang, I. M. Ross, "Zero Propellant Maneuver Guidance," IEEE Control Systems Magazine, Vol. 29, Issue 5, October 2009, pp. 53-73.

[24] S. Bhatt, N. Bedrossian, K. Longacre and L. Nguyen, "Optimal Propellant Maneuver Flight Demonstrations on ISS," AIAA Guidance, Navigation, and Control Conference, August 1922, 2013, Boston, MA. AIAA 2013-5027.

[25] N. Bedrossian, M. Karpenko, and S. Bhatt, "Overclock My Satellite: Sophisticated Algorithms Boost Satellite Performance on the Cheap," IEEE Spectrum Magazine, Vol. 49, No. 11, 2012, pp. 54-62.

[26] M. Karpenko, S. Bhatt, N. Bedrossian, and I. M. Ross, "Flight Implementation of ShortestTime Maneuvers for Imaging Satellites," Journal of Guidance Control and Dynamics, Vol.37, No.4, 2014, pp.1069-1079.

[27] G. Minelli, M. Karpenko, I. M. Ross and J. Newman, "Autonomous Operations of LargeScale Satellite Constellations and Ground Station Networks," AAS/AIAA Astrodynamics Specialist Conference, August 20 - 24, 2017 Stevenson, WA. AAS-17-761

[28] N. Bedrossian, S. Bhatt, M. Lammers and L. Nguyen, "Zero Propellant Maneuver: Flight Results for $180^{\circ}$ ISS Rotation," 20th International Symposium on Space Flight Dynamics, September 24-28, 2007, Annapolis, MD, NASA/CP-2007-214158.

[29] H. Yan, Q. Gong, C. Park, I. M. Ross, and C. N. D’Souza, "High Accuracy Trajectory Optimization for a Trans-Earth Lunar Mission," Journal of Guidance, Control and Dynamics, Vol. 34, No. 4, 2011, pp. 1219-1227.

[30] I. M. Ross, Q. Gong, M. Karpenko and R. J. Proulx, "Scaling and Balancing for HighPerformance Computation of Optimal Controls," Journal of Guidance, Control and Dynamics, to appear. 
[31] I. M. Ross, M. Karpenko and R. J. Proulx, “The Million Point Computational Optimal Control Challenge," SIAM Conference on Control and its Applications, MS24, July 10-12, 2017, Pittsburgh, PA.

[32] I. M. Ross, M. Karpenko and R. J. Proulx, "A Nonsmooth Calculus for Solving Some GraphTheoretic Control Problems," IFAC-PapersOnLine 49-18, 2016, pp. 462-467

[33] I. M. Ross, R. J. Proulx, J. M. Greenslade and M. Karpenko, "Dynamic Optimization for Satellite Image Collection," Advances in the Astronautical Sciences: Spaceflight Mechanics, Vol. 158, AAS 16-260, 2016, pp. 199-218

[34] J. S. Hesthaven, "Integration Preconditioning Of Pseudospectral Operators. I. Basic Linear Operators," SIAM Journal of Numerical Analysis, Vol. 35, No. 4, pp. 1571-1593, 1998.

[35] E. M. E. Elbarbary, "Integration Preconditioning Matrix for Ultraspherical Pseudospectral Operators," SIAM Journal of Scientific Computaton, Vol. 28, No. 3, pp. 1186-1201, 2006.

[36] L.-L Wang, M. D. Samson and X. Zhao, "A Well-Conditioned Collocation Method Using a Pseudospectral Integration Matrix," SIAM Journal of Scientific Computaton, Vol. 36, No. 3, pp. A907-A929, 2014.

[37] S. Olver and A. Townsend, "A Fast and Well-Conditioned Spectral Method," SIAM Review, Vol. 55, No. 3, pp. 462-489, 2013.

[38] K. Du, "On Well-Conditioned Spectral Collocation and Spectral Methods by the Integral Reformulation," SIAM Journal of Scientific Computaton, Vol. 38, No. 5, pp. A3247-A3263, 2016.

[39] I. M. Ross, A Primer on Pontryagin's Principle in Optimal Control, Second Edition, Collegiate Publishers, San Francisco, CA, 2015.

[40] J. M. Longuski, J. J. Guzmán and J. E. Prussing, Optimal Control with Aerospace Applications, Springer, New York, N.Y., 2014.

[41] F. Clarke, Functional Analysis, Calculus of Variations and Optimal Control, Springer-Verlag, London, 2013; Ch. 22.

[42] F. Fahroo and I. M. Ross, "Advances in Pseudospectral Methods for Optimal Control," AIAA Guidance, Navigation, and Control Conference, AIAA Paper 2008-7309, Honolulu, Hawaii, August 2008.

[43] T. Tao, An Epsilon of Room, Vol. I, American Mathematical Society, Providence, RI, 2010, Ch. 1 .

[44] Q. Gong, I. M. Ross and F. Fahroo, "Pseudospectral Optimal Control On Arbitrary Grids," AAS Astrodynamics Specialist Conference, AAS-09-405, 2009.

[45] Q. Gong, I. M. Ross and F. Fahroo, "Spectral and Pseudospectral Optimal Control Over Arbitrary Grids," Journal of Optimization Theory and Applications, vol. 169, no. 3, pp. 759783, 2016. 
[46] W. W. Hager, "Runge-Kutta Discretizations of Optimal Control Problems," System Theory: Modeling Analysis and Control, T. E. Djaferis and I. C. Schick (Eds), Springer US, pp. 233244, 2000.

[47] I. M. Ross, Q. Gong and P. Sekhavat, "Low-Thrust, High-Accuracy Trajectory Optimization," Journal of Guidance, Control and Dynamics, Vol. 30, No. 4, pp. 921-933, 2007.

[48] I. M. Ross, Q. Gong and P. Sekhavat, "The Bellman Pseudospectral Method," AIAA/AAS Astrodynamics Specialist Conference and Exhibit, Honolulu, Hawaii, AIAA-2008-6448, August 18-21, 2008.

[49] D. Kosloff and H. Tal-Ezer, "A Modified Chebyshev Pseudospectral Method With An $\mathcal{O}\left(N^{-1}\right)$ Time Step Restriction," Journal of Computational Physics, Vol. 104, No. 2, 1993, pp. 457-469.

[50] I. M. Ross, F. Fahroo and J. Strizzi, "Adaptive Grids for Trajectory Optimization by Pseudospectral Methods," AAS/AIAA Spaceflight Mechanics Conference, Paper No. AAS 03-142, Ponce, Puerto Rico, 9-13 February 2003.

[51] G. G. Lorentz and K. L. Zeller, "Birkhoff Interpolation,” SIAM Journal of Numerical Analysis, Vol. 8, No. 1, pp. 43-48, 1971.

[52] I. J. Schoenberg, “On Hermite-Birkhoff Interpolation,” Journal of Mathematical Analysis and Applications, Vol. 16, No. 3, pp. 538-543, 1966.

[53] W. F. Finden, "An Error Term and Uniqueness for Hermite-Birkhoff Interpolation Involving Only Function Values and/or First Derivative Values," Journal of Computational and Applied Mathematics, Vol. 212, No. 1, pp. 1-15, 2008.

[54] N. Koeppen, "Well-Conditioned Pseudospectral Optimal Control Methods and Their Applications," M.S. Thesis in Applied Mathematics, Naval Postgraduate School, June 2018.

[55] F. Fahroo and I. M. Ross, "Pseudospectral Methods for Infinite-Horizon Nonlinear Optimal Control Problems," Proceedings of the AIAA Guidance, Navigation and Control Conference, San Francisco, CA, August 15-18, 2005.

[56] F. Fahroo and I. M. Ross, "Pseudospectral Methods for Infinite-Horizon Optimal Control Problems," Journal of Guidance, Control and Dynamics, Vol. 31, No. 4, pp. 927-936, 2008.

[57] B. A. Conway, "The Problem of Space Trajectory Optimization," Space Trajectory Optimization, B. A. Conway (ed.), Cambridge Aerospace Series, Vol. 29, 2010, pp. 1-15. 